\title{
Rural Status, Suicide Ideation, and Telemental Health: Risk Assessment in a Clinical Sample
}

Kevin R. Tarlow, PhD; ${ }^{1}$ Tina A. Johnson, MPH $;{ }^{1} \&$ Carly E. McCord, $\mathrm{PhD}^{1}$

${ }^{1}$ School of Public Health, Texas A\&M University, College Station, Texas

Citation: Tarlow, K. R., Johnson, T. A., \& McCord, C. E. (in press). Rural status, suicide ideation, and telemental health: Risk assessment in a clinical sample. Journal of Rural Health.

Disclosures: The authors report no conflicts of interest.

Funding: This work was supported by Medicaid Section 1115(a) Demonstration, "Texas Healthcare Transformation and Quality Improvement Program" (Project Number 11-W00278/6).

For further information, contact: Carly E. McCord, $\mathrm{PhD}$, Telehealth Counseling Clinic, 4225 TAMU, College Station, TX, 77803-4225; cmccord@sph.tamhsc.edu.

Key Words: health disparities; rural mental health, suicide, telehealth; telepsychology 


\title{
Rural Status, Suicide Ideation, and Telemental Health: Risk Assessment in a Clinical Sample
}

\begin{abstract}
Objective: Individuals living in rural areas die by suicide at higher rates than those living in metropolitan areas. Telemental health interventions provide rural residing individuals with access to needed care. Identifying telemental health clients at risk for suicide is an important task for clinicians and policymakers. The present study evaluated to what degree rural status and other demographic variables predicted suicide ideation in clients seeking services at a telemental health clinic.
\end{abstract}

Methods: Study participants included 457 low-income clients residing in the medically underserved, geographically diverse Brazos Valley region of Texas. Clients completed the Patient Health Questionnaire (PHQ) during their initial counseling appointment, which assessed depression severity and suicide ideation in the 2 weeks prior to assessment.

Results: Suicide ideation was common among telemental health clients, with approximately $40 \%$ of clients in all demographic groups reporting some recent thoughts of suicide. Rates of suicide ideation did not significantly differ by geographic designation (ie, rural/metropolitan status), gender, or race/ethnicity. However, depression was a strong predictor of recent suicide ideation.

Conclusions: Telemental health programs can effectively connect clinicians with rural residing clients who are otherwise isolated from health care services. However, clinicians working in high-need, historically underserved areas should be prepared to encounter a high prevalence of 
suicide ideation and depression. In these regions, clinical and diagnostic features may be better indicators of suicide ideation than demographic variables.

Key Words: health disparities; rural mental health, suicide, telehealth; telepsychology 
Rural areas have significantly higher suicide mortality than urban areas, and the urban-rural disparity in suicide rate has increased in the past decade ${ }^{1,2}$ The causes of this disparity are not well understood. Mental disorder diagnosis is a strong predictor of suicide, but mental disorders occur at about the same rates in rural and urban communities. ${ }^{3-5}$ Suicide ideation is an important antecedent to suicide mortality; ${ }^{6,7}$ however, studies in Europe and Australia found that rural status does not predict differences in suicide ideation. ${ }^{8,9}$ Urban and rural communities also exhibited no differences in suicide attempts and previous history of suicide. ${ }^{10,11}$ This suggests other confounding variables may explain the rural-urban suicide gap. Rural regions have always had disproportionately high rates of poverty in the United States; ${ }^{12}$ low socioeconomic status and some of its correlates (such as low mental health service utilization and unemployment) are predictors of death by suicide. ${ }^{10,13}$ Firearms are also more accessible to rural residing individuals, and disproportionate access to these highly lethal means of suicide may further explain disparities. $^{14}$

In addition to rural status, gender, race/ethnicity, and age are important demographic predictors of suicide risk. ${ }^{15}$ Men are more likely to die by suicide, though women are more likely to attempt suicide. Whites die by suicide at higher rates than other racial/ethnic groups. Older individuals are also at the greatest risk of suicide. Demographic risk factors are generally cumulative, such that older white men have the highest average suicide rate. However, demographics tend to be weaker predictors of suicide ideation than suicide mortality. ${ }^{16,17}$ Geographic and demographic factors also demonstrate complex and heterogeneous interactions across regions, cultures, and lifespan. ${ }^{18}$ Suicide risk is further complicated by the accumulation of psychiatric diagnoses. Suicide risk increases with an individual's total number of mental 
disorder diagnoses, though depression is the most common disorder in people who die by suicide. $^{19,20}$

Telehealth — the use of telecommunications technology to deliver health care services from a distance - is an effective and efficient option for delivering mental health care services to at-risk rural populations who otherwise have few options for treatment. ${ }^{21,22}$ However, the use of telehealth with clients at risk of suicide raises clinical, ethical, and legal concerns. ${ }^{23}$ Clinicians must assess risk without the rich clinical data available during a typical in-person encounter. Emergency interventions, such as involuntary hospitalization, are difficult when a clinician is not physically present with a suicidal client. Clinicians may also assume increased liability for at-risk clients in the event of a technology failure that impedes risk assessment and safety planning. It is therefore crucial for telemental health care providers to understand how best to assess suicide risk and, when necessary, intervene effectively from a distance to ensure their clients' safety.

One aspect of suicide risk assessment relevant to this study is understanding how suicide ideators become suicide attempters. Most people who attempt suicide had previous thoughts of suicide; however, most people who contemplate suicide do not attempt. ${ }^{6}$ The pathway from ideation to attempting is complex, but research suggests depression is a strong predictor of ideation onset, though anxiety/agitation (as characterized by posttraumatic stress disorder and panic) and impulse control problems (as in conduct and substance use disorders) better predict which ideators progress to suicide attempts. ${ }^{6,24}$ Due to the developing nature of rural suicide research, the present study focuses on the first half of the ideation-to-attempt pathway, ie, the urban-rural disparities in suicide ideation in a clinical sample, though future research should similarly examine disparities in the progression of suicide ideation and behavior. 
The goal of the present study is to clarify the geographic and demographic risk factors that predict suicide ideation in a telemental health clinical sample. Rural residing individuals are understood to be at higher risk of death by suicide compared to those in metropolitan areas, but it is unclear if and how suicide ideation, gender, race/ethnicity, and age contribute to this pattern. As telemental health services are increasingly deployed to rural communities, clinicians would benefit from a better understanding of the relationship between rural status and suicide ideation.

\section{Methods}

\section{Participants}

The present study analyzed archival data from a telemental health clinic located in the Texas Brazos Valley - a geographically diverse region in the eastern half of the state. Participants included all 457 adults who received services at the clinic between 2010 and 2017. The Brazos Valley is a medically underserved region with higher than expected rates of depression, particularly among women and black residents. ${ }^{3}$ The telemental health clinic provides free counseling services to mostly low-income and uninsured clients. Five out of 7 Brazos Valley counties were served by the telemental health clinic, which uses a "hub and spoke" model in which clients travel to nearby access points in their home counties to receive services from centrally located service providers. Counseling services are offered via videoconference technology and/or phone by doctoral student counselors enrolled in APA accredited counseling/clinical psychology programs. The 5 counties served by the telemental health clinic span 3 of the urban-rural classifications provided by the Centers for Disease Control and Prevention: 1 county (Brazos: 194,851 residents) is classified as "small metropolitan" (includes a city of 50,000 - 249,999 residents), 1 county (Washington: 33,718) is classified as 
"micropolitan" (includes a city of 10,000 - 49,999 residents), and 3 counties (Grimes: 26,604;

Leon: 16,801; Madison: 13,664) are classified as "noncore" (does not include a city of 10,000 or

more residents). ${ }^{25}$ Demographic information for participants, grouped by urban-rural classification, are presented in Table 1.

\section{INSERT TABLE 1 HERE}

\section{Measures}

Measures included information gathered during the clinic's routine client intake process. This included demographic information and information about clients' county of residence. In their first session, clients also completed the Patient Health Questionnaire (PHQ). ${ }^{26}$ The PHQ was administered to all clients primarily for treatment planning and outcome monitoring purposes. Nine PHQ items assess depression severity, and they may be used together as a brief depression screener, the PHQ-9. One of the PHQ-9 items assesses suicide ideation, which was the outcome variable of interest for the present study. Any reported suicide ideation in the 2 weeks prior to intake was scored as a 1 , whereas a 0 for suicide ideation indicated no thoughts of suicide in the 2 weeks prior to intake. The PHQ is a widely used license-free mental health assessment, and scores of the PHQ-9 depression screener have demonstrated excellent internal validity $(\alpha=0.89)$ and diagnostic validity (sensitivity: $88 \%$; specificity: $88 \%$ ). ${ }^{27}$ The first 8 items of the PHQ-9, excluding the suicide ideation item, may be used independently for assessment and research purposes, and together comprise the PHQ-8. Scores of the PHQ-8 have demonstrated similar validity to the PHQ-9. ${ }^{28}$ In addition to demographic predictors, a probable depression diagnosis was recorded for clients with a PHQ-8 score of $\geq 10$, consistent with recommended diagnostic cutoffs. (PHQ-8 rather than PHQ-9 scores were used because the suicide item served as the 
dependent variable; thus, separating probable depression diagnosis and suicide ideation maintained statistical independence of outcome and predictor.)

\section{Statistical Analyses}

Prevalence of suicide ideation was assessed using PHQ-9 response. Because suicide ideation is a binary outcome, logistic regression was used to evaluate how well geographic, demographic, and clinical risk factors predicted suicide ideation in the clinical samples. The 3 models were, in order of increasing complexity: (1) suicide ideation = urban-rural classification, (2) suicide ideation $=$ urban-rural classification + gender + race/ethnicity + age, and $(3)$ suicide ideation $=$ urban-rural classification + gender + race/ethnicity + age + probable depression diagnosis (via PHQ-8). Strength of association was measured via odds ratio (OR), a ratio of the odds of suicide ideation in the interest group over the odds of suicide ideation in the reference group. An OR greater than 1 indicates a positive association, an OR less than 1 indicates a negative association, and an OR equal to 1 indicates no association. In addition, 95\% confidence intervals were created for each analysis, and the Wald test (z-test) was used to test the significance of associations for individual predictors. Likelihood ratio tests were also used to determine the relative goodness of the logistic models' fit. All analyses were conducted with R version $3.4 .^{29}$

\section{Ethical Approval}

The study was approved by an Institutional Review Board (\#IRB2018-0043M). All participants provided written informed consent to participate in research.

\section{Results}

Prevalence of suicide ideation and results of the 3 logistic regression models, including odds ratios, are presented in Table 2. The prevalence of suicide ideation was consistent across 
geographic and demographic groups. The overall prevalence of suicide ideation was $43 \%$. In other words, 195 out of 457 clients stated they had "thoughts that [I] would be better off dead or of hurting [myself] in some way" during the 2 weeks prior to their first telemental health session. To put this high rate of suicide ideation into context, the 1-year prevalence of suicide ideation in a national survey was $4 \% .{ }^{30}$ Out of the 195 clients with suicidal thoughts, 110 (or $24 \%$ of all clients) reported ideation for "several days" during the past 2 weeks, $44(10 \%)$ reported ideation for "more than half the days," and $41(9 \%)$ reported ideation "nearly every day."

\section{INSERT TABLE 2 HERE}

Urban-rural classification was not a statistically significant predictor of suicide ideation in Model 1 (which included only urban-rural classification), Model 2 (which added gender, race/ethnicity, and age), or Model 3 (which added probable depression diagnosis). The demographic predictors were also not statistically significant predictors of suicide ideation in Model 2 or Model 3. Probable depression diagnosis, as determined by a PHQ-8 score of $\geq 10$, was the only statistically significant predictor, $\mathrm{OR}=4.16,95 \% \mathrm{CI}(2.57-6.94), P<.001$. A likelihood ratio test failed to detect a statistically significant difference between a null model and Model $1(P=.503)$ or between a null model and Model $2(P=.932)$. Model 1 and Model 2 were also not statistically significantly different $(P=.957)$. However, Model 3's fit was improved over Model $2(P<.001)$. A likelihood ratio test indicated Model 3 did not yield a better fit than a logistic model with only the probable depression diagnosis predictor $(P=.586)$.

\section{Discussion}

The goal of the present study was to determine how rural status predicted suicide ideation in a sample of telemental health clients. Telehealth is a promising modality for treating high-risk 
individuals who live in rural, historically underserved communities. Increased rates of suicide mortality in rural areas suggest that understanding rural suicide is a high priority for mental health workers who wish to better utilize telehealth interventions in geographically remote regions, such as the rural Texas Brazos Valley.

Rural status did not predict observed differences in suicide ideation in the 3 models tested. Though suicide ideation is an important antecedent to death by suicide, this result is consistent with previous studies which found no urban-rural differences in suicide ideation, despite documented urban-rural differences in suicide mortality. ${ }^{8,9}$ Similarly, gender, age, and race/ethnicity did not predict differences in suicide ideation.

The only statistically significant predictor of suicide ideation was a probable depression diagnosis, as determined by a PHQ-8 score of $\geq 10$. Individuals diagnosed with depression had approximately 4 times the odds of reporting recent suicide ideation compared to individuals without a probable depression diagnosis. Stated another way, $51 \%$ of individuals with a probable depression diagnosis at intake reported thoughts of suicide in the 2 weeks prior to treatment. By comparison, only $21 \%$ of individuals without a probable depression diagnosis reported recent suicide ideation.

These results suggest that clients' diagnostic presentations are more likely to help telemental health clinicians anticipate and detect suicide ideation than geographic or demographic variables. This may be particularly true for clients in chronically underserved regions or communities, who present to treatment with substantial, and often co-occurring, concerns. High levels of psychological distress - likely exacerbated by poverty and poor access to care - may be stronger determinants of suicide ideation than rural status, gender, age, and 
race/ethnicity effects, which are useful predictors in public health surveillance of nonclinical populations.

The present study illustrates how clinical and diagnostic factors may outweigh the usefulness of demographic factors in a high-need, high-severity sample. For example, the average PHQ-9 depression severity score of participants in the present study was 14.4, which falls above the 98th percentile of a general population sample, meaning that the average client served by the telemental health clinic had depression symptoms as severe or more severe than $98 \%$ of other people. ${ }^{31,32}$ In addition, $74 \%$ of telemental health clients in the present study had PHQ-9 scores of 10 or more, indicating a likely depression diagnosis. To put this high rate of depression into context, only $5 \%$ of respondents in the general population score at or above a PHQ-9 score of 10, yet three-fourths of clients receiving telemental health care in the present study achieved this criterion. Put simply, the untreated mental health needs of this low-income, underserved clinical sample are, for both the average client and clinician, more urgent than differences due to age, gender, or race/ethnicity. That said, cultural differences inform how symptoms of depression and other disorders are experienced and communicated. ${ }^{33,34}$ Multicultural competence is therefore essential for accurate diagnosis and risk assessment. For example, clinicians working with rural populations should be informed about rural suicide risk factors, such as increased access to firearms and other lethal means of self-injury, and they should evaluate these risks accordingly.

\section{Conclusions}

Telemental health interventions have the potential to reach individuals historically isolated from the mental health care system, either by geographic, economic, or other barriers. Rural residing 
individuals may disproportionately benefit from telehealth due to the frequent shortage of service providers in these areas. Suicide risk assessment is an important component of all mental health care, though it is complicated when emergent safety concerns must be managed at a distance. The present study demonstrated that probable depression diagnosis was the strongest predictor of suicide ideation in this high-need clinical sample when compared to geographic and demographic variables. Future research should determine what other clinical and diagnostic factors inform suicide risk assessment in order to better equip telemental health care providers, particularly those working with rural residing clients. Further study of geographic disparities in diagnostic factors may also clarify the disproportionate progression from suicide ideation to lethal attempts in rural populations. Disorders characterized by anxiety/agitation (eg, posttraumatic stress disorder) and impulse control problems (eg, conduct and substance use disorders) deserve additional attention, as these symptoms may facilitate the ideation-to-attempt pathway. ${ }^{6,24}$ If suicide attempts are found to occur at similar rates in rural and urban areas, then access to lethal means (eg, firearms) may be the most useful indicator of mortality risk.

This study has other limitations, in addition to its limited focus on suicide ideation (and not the progression of ideation to suicide attempts). Participants were constrained to a relatively small geographic region, and rural communities are known to be diverse in terms of their culture, ${ }^{35}$ resources, ${ }^{12}$ and health status. ${ }^{18}$ The study also did not include comparisons with urban residing individuals living in large metropolitan areas (the most populous county served by the clinic was designated "small metropolitan"); it is plausible that urban-rural differences in suicide ideation would be detected if noncore (ie, rural) county residents were compared with individuals in major urban population centers. These results should therefore be generalized with care; however, it was notable that the present study failed to detect urban-rural differences in suicide 
ideation, similar to studies of other regions. ${ }^{89}$ This study used a clinical sample of participants, so on the one hand participants were expected to have more severe mental health concerns than a community sample; however, because all participants were actively seeking mental health care services, they may have been disproportionately motivated and willing to disclose psychological problems. Due to the archival nature of this study, analyses were also limited to the self-report clinical outcome measures used in the routine care of all clients served by the clinic (eg, PHQ-9). Diagnoses provided by clinician evaluation, rather than probable diagnoses determined by PHQ cutoff scores, would improve the validity of future research. In addition, suicide-specific instruments would better discriminate between suicide ideation, intensity, and behavior. ${ }^{36}$ 


\section{References}

1. Kegler, SR, Stone, DM, Holland, KM. Trends in suicide by level of urbanization United States, 1999-2015. MMWR. Morbidity and Mortality Weekly Reports. 2017;66:270-273.

2. Hirsch JK, Cukrowicz KC. Suicide in rural areas: An updated review of the literature. Journal of Rural Mental Health. 2014;38(2):65-78.

3. Brossart DF, Wendel ML, Elliott TR, Cook HE, Castillo LG, Burdine JN. Assessing depression in rural communities. Journal of Clinical Psychology. 2013;69(3):252-263.

4. Gamm LG, Stone S, Pittman S. Mental health and mental disorders-A rural challenge: A literature review. In: Gamm L, Hutchinson LL, Dabney BJ, Dorsey AM, eds. Rural Healthy People 2010: A Companion Document to Healthy People 2010, Volume 2. College Station, TX: The Texas A\&M University System Health Science Center, School of Rural Public Health, Southwest Rural Health Research Center; 2003:97-113.

5. McCall-Hosenfeld JS, Mukherjee S, Lehman EB. The prevalence and correlates of lifetime psychiatric disorders and trauma exposures in urban and rural settings: Results from the national comorbidity survey replication (NCS-R). PLOS ONE. 2014;9(11):1-11.

6. May AM, Klonsky ED. What distinguishes suicide attemptors from suicide ideators? Clinical Psychology: Science and Practice. 2016;32(1):5-20.

7. Nock MK, Millner AJ, Joiner TE, et al. Risk factors for the transition from suicide ideation to suicide attempt: Results from the Army Study to Assess Risk and Resilience in Servicemembers (Army STARRS). Journal of Abnormal Psychology. 2018;127(2):139-149. 
8. Casey PR, Dunn G, Kelly BD, et al. Factors associated with suicidal ideation in the general population: Five-centre analysis from ODIN study. British Journal of Psychiatry. 2006;189(5):410-415.

9. Inder KJ., Handley TE, Johnston A, et al. Determinants of suicidal ideation and suicide attempts: Parallel cross-sectional analyses examining geographical location. BioMed Central Psychiatry. 2014;14(208):1-20.

10. Hempstead K. The geography of self-injury: Spatial patterns in attempted and completed suicide. Social Science \& Medicine. 2006; 62(12):3186-3196.

11. Taylor R, Page A, Morrell S, Harrison J, Carter G. Social and psychiatric influences on urban-rural differentials in Australian suicide. Suicide and Life-Threatening Behavior. 2005; 35(3):277-290.

12. Tickamyer A, Warlick J, Sherman J, eds. Rural poverty in the United States. New York, NY: Columbia University Press; 2017.

13. Denney JT, Rogers RG, Krueger PM, Wadsworth T. Adult suicide mortality in the United States: Marital status, family size, socioeconomic status, and differences by sex. Social Science Quarterly. 2009;90(5):1167-1185.

14. Nestadt PS, Triplett P, Fowler DR, Mojtabai R. Urban-rural differences in suicide in the state of Maryland: The role of firearms. AJPH Rural Health. 2017;107(10):1548-1553.

15. Joiner TE. Why People Die by Suicide. Cambridge, MA: Harvard University Press; 2005.

16. Kessler RC, Berglund P, Demler O, Jin R, Merikangas KR,Walters EE. Lifetime prevalence and age-of-onset distributions of DSM-IV disorders in the national comorbidity survey replication. Arch Gen Psychiatry. 2005;62(6):593-602. 
17. Weissman MM, Bland RC, Canino GJ, et al. Prevalence of suicidal ideation and suicide attempts in nine countries. Psychological Medicine. 1999;29(1):9-17.

18. Meit M, Knudson A, Gilbert T, et al. The 2014 Update of the Rural-Urban Chartbook. Bethesda, MD: The Rural Health Reform Policy Research Center; 2014. Available at: https://ruralhealth.und.edu/projects/health-reform-policy-research-center/pdf/2014-ruralurban-chartbook-update.pdf. Accessed February 19, 2018.

19. Hawton K, Casañas I Comabella C, Haw C, Saunders K. Risk factors for suicide in individuals with depression: A systematic review. Journal of Affective Disorders. 2013; $147(1-3): 17-28$.

20. Kessler RC, Borges G, Walters EE. Prevalence of and risk factors for lifetime suicide attempts in the National Comorbidity Survey. Archives of General Psychiatry. $1999 ; 56: 617-626$.

21. Jong M. Managing suicides via videoconferencing in a remote northern community in Canada. International Journal of Circumpolar Health. 2004;63(4):422-428.

22. Shore JH, Brooks E, Savin DM, Manson SM, Libby AM. An economic evaluation of telehealth data collection with rural populations. Psychiatric Services. 2007;58(6):830835.

23. Goldeski L, Nieves JE, Darkins A, Lehmann L.VA telemental health: Suicide assessment. Behavioral Sciences and the Law. 2008;26(3):271-286.

24. Nock MK, Hwang I, Sampson NA, Kessler RC. Mental disorders comorbidity, and suicidal behavior: Results from the National Comorbidity Survey Replication. Molecular Psychiatry. 2010;15:868-876. 
25. Ingram DD, Franco SJ. 2013 NCHS urban-rural classification scheme for counties. Vital and Health Statistics.2013;2(166):1-74.

26. Spitzer RL, Kroenke K, Williams JB. Validation and utility of a self-report version of PRIME-MD — the PHQ primary care study. JAMA. 1999; 282(18):1737-1744.

27. Kroenke K, Spitzer RL, Williams, JB. The PHQ-9: Validity of a brief depression severity measure. Journal of General Internal Medicine. 2001;16(9):606-616.

28. Kroenke K, Strine TW, Spitzer RL, Williams JB, Berry JT, Mokdad AH. The PHQ-8 as a measure of current depression in the general population. Journal of Affective Disorders. 2009;114(1-3):163-173.

29. R: The R Project for Statistical Computing [computer program].Version 3.4. [software]. Vienna, Austria: R Foundation for Statistical Computing. http://www.R-project.org/. Accessed November 3, 2017.

30. Substance Abuse and Mental Health Services Administration. Key substance use and mental health indicators in the United States: Results from the 2016 National Survey on Drug Use and Health (HHS Publication No. SMA 17-5044, NSDUH Series H-52). Rockville, MD: Center for Behavioral Health Statistics and Quality, Substance Abuse and Mental Health Services Administration; 2017.

31. Kocalevent RD, Hinz A, Brahler E. Standardization of the depression screener patient health questionnaire (PHQ-9) in the general population. General Hospital Psychiatry. $2013 ; 35(5): 551-555$.

32. Shim RS, Baltrus P, Ye J, Rust G. Prevalence, treatment, and control of depressive symptoms in the United States: Results from the National Health and Nutrition 
Examination Survey (NHANES), 2005-2008. Journal of the American Board of Family Medicine. 2011;24(1):33-38.

33. Ridley CR, Li LC, Hill CL. Multicultural assessment: Reexamination, reconceptualization, and practical application. The Counseling Psychologist. 1998;26(6):827-910.

34. Suzuki LA, Ponterotto JG, Meller PJ. Handbook of Multicultural assessment: Clinical, Psychological, and Educational Applications. 3rd ed. San Francisco, CA: John Wiley \& Sons, Inc.; 2008.

35. Slama K. Rural culture is a diversity issue. Minnesota Psychologist. 2004;53(1):9-12.

36. Posner K, Grown GK, Stanley B, et al. The Columbia-Suicide Severity Rating Scale: Initial validity and internal consistency findings from three multisite studies with adolescents and adults. American Journal of Psychiatry. 2011;168(12):1266-1277. 
Table 1. Demographic Information of Participants by Urban-Rural Classification $(n=457)$

\begin{tabular}{|c|c|c|c|}
\hline & \multicolumn{3}{|c|}{ Urban-Rural Classification } \\
\hline & Small Metropolitan $^{\mathrm{a}}$ & Micropolitan $^{\mathrm{b}}$ & Noncore $^{\mathrm{c}}$ \\
\hline Female & 135 & 44 & 155 \\
\hline Male & 58 & 17 & 48 \\
\hline White & 104 & 39 & 138 \\
\hline Black & 36 & 14 & 34 \\
\hline Hispanic/Latinx & 38 & 5 & 26 \\
\hline$>1$ race or Other & 15 & 3 & 5 \\
\hline Age: M (SD) & $38(14)$ & $43(15)$ & $43(13)$ \\
\hline
\end{tabular}

${ }^{a}$ Brazos County; ${ }^{b}$ Washington County; ${ }^{c}$ Grimes, Leon, and Madison Counties 
Table 2. Logistic Regression Models of Suicide Ideation Predictors $(n=457)$

\begin{tabular}{|c|c|c|c|c|c|c|c|c|}
\hline & \multirow[b]{2}{*}{$\begin{array}{c}\text { No Suicide Ideation } \\
(\%)\end{array}$} & \multirow[b]{2}{*}{$\begin{array}{c}\text { Suicide Ideation } \\
(\%)\end{array}$} & \multicolumn{2}{|c|}{ Model $1^{\mathrm{a}}$} & \multicolumn{2}{|c|}{ Model 2 ${ }^{\mathrm{b}}$} & \multicolumn{2}{|c|}{$\operatorname{Model~} 3^{c}$} \\
\hline & & & $\begin{array}{c}\mathrm{OR} \\
(95 \% \mathrm{CI})\end{array}$ & $P$ & $\begin{array}{c}\mathrm{OR} \\
(95 \% \mathrm{CI})\end{array}$ & $P$ & $\begin{array}{c}\mathrm{OR} \\
(95 \% \mathrm{CI})\end{array}$ & $P$ \\
\hline \multicolumn{9}{|l|}{ Urban-Rural Classification } \\
\hline Sm Metro & $\begin{array}{c}108 \\
(0.56)\end{array}$ & $\begin{array}{c}85 \\
(0.44)\end{array}$ & Reference & - & Reference & - & Reference & - \\
\hline Micro & $\begin{array}{c}32 \\
(0.52)\end{array}$ & $\begin{array}{c}29 \\
(0.48)\end{array}$ & $\begin{array}{c}1.15 \\
(0.64-2.05)\end{array}$ & .63 & $\begin{array}{c}1.17 \\
(0.65-2.11)\end{array}$ & .59 & $\begin{array}{c}1.09 \\
(0.58-2.02)\end{array}$ & .79 \\
\hline Noncore & $\begin{array}{c}122 \\
(0.60) \\
\end{array}$ & $\begin{array}{c}81 \\
(0.40) \\
\end{array}$ & $\begin{array}{c}0.84 \\
(0.56-1.25) \\
\end{array}$ & .40 & $\begin{array}{c}0.87 \\
(0.58-1.30) \\
\end{array}$ & .49 & $\begin{array}{c}0.77 \\
(0.50-1.18) \\
\end{array}$ & .23 \\
\hline Gender & & & - & - & & & & \\
\hline Male & $\begin{array}{c}72 \\
(0.58)\end{array}$ & $\begin{array}{c}51 \\
(0.41)\end{array}$ & - & - & Reference & - & Reference & - \\
\hline Female & $\begin{array}{c}190 \\
(0.57)\end{array}$ & $\begin{array}{c}144 \\
(0.43)\end{array}$ & - & - & $\begin{array}{c}1.10 \\
(0.72-1.70)\end{array}$ & .64 & $\begin{array}{c}1.04 \\
(0.66-1.64)\end{array}$ & .85 \\
\hline Race/Ethnicity & & & - & - & & & & \\
\hline White & $\begin{array}{c}164 \\
(0.58)\end{array}$ & $\begin{array}{l}117 \\
(0.42)\end{array}$ & - & - & Reference & - & Reference & - \\
\hline Black & $\begin{array}{c}46 \\
(0.55)\end{array}$ & $\begin{array}{c}38 \\
(0.45)\end{array}$ & - & - & $\begin{array}{c}1.14 \\
(0.69-1.86)\end{array}$ & .61 & $\begin{array}{c}1.17 \\
(0.70-1.96)\end{array}$ & .55 \\
\hline Hispanic & $\begin{array}{c}40 \\
(0.58)\end{array}$ & $\begin{array}{c}29 \\
(0.42)\end{array}$ & - & - & $\begin{array}{c}0.99 \\
(0.57-1.69)\end{array}$ & .97 & $\begin{array}{c}1.23 \\
(0.68-2.20)\end{array}$ & .49 \\
\hline$>1$ race or other & $\begin{array}{c}11 \\
(0.48)\end{array}$ & $\begin{array}{c}12 \\
(0.52)\end{array}$ & - & - & $\begin{array}{c}1.21 \\
(0.50-2.93)\end{array}$ & .66 & $\begin{array}{c}1.41 \\
(0.55-3.66)\end{array}$ & .47 \\
\hline Age & - & - & - & - & $\begin{array}{c}1.00 \\
(0.98-1.01) \\
\end{array}$ & .52 & $\begin{array}{c}0.99 \\
(0.98-1.00) \\
\end{array}$ & .23 \\
\hline No Depression $(\mathrm{PHQ}-8<10)$ & $\begin{array}{c}98 \\
(0.79)\end{array}$ & $\begin{array}{c}26 \\
(0.21)\end{array}$ & - & - & - & - & Reference & - \\
\hline Depression (PHQ-8 $\geq 10$ ) & $\begin{array}{c}164 \\
(0.49)\end{array}$ & $\begin{array}{c}169 \\
(0.51)\end{array}$ & - & - & - & - & $\begin{array}{c}4.16 \\
(2.57,6.94)\end{array}$ & $<.001$ \\
\hline
\end{tabular}

${ }^{\mathrm{a}}$ Urban-rural only model; ${ }^{\mathrm{b}}$ Urban-rural, gender, age, and race/ethnicity model; ${ }^{\mathrm{c}}$ Urban-rural, gender, age, race/ethnicity, and depression model 